to the present-day osteopathic medical students.

If we are lax in our commitment to our profession's continued viability, we will have failed not only the next generation of osteopathic physicians, but also our teaching institutions, our patients, ourselves, and our country. If we are prohibited from participating in private-sector healthcare programs, our licenses will be of little value.

The struggle to retain our practice privileges seems to have begun-again. If we lose this fight, the osteopathic medical philosophy could be lost forever.

RANDOLPHE ROULIER, DO

Chairman, Department of Surgery

Parkview Hospital

Toledo, Ohio

\section{Possible suprascapular nerve involvement in injured supraspinatus muscle}

\section{To the Editor:}

In the article "Shoulder pain and repetition strain injury to the supraspinatus muscle: Etiology and manipulative treatment"(JAOA 1989;89:1037-1045), Dr Jacobson and his colleagues did not indicate any possible involvement of the suprascapular nerve. Suprascapular nerve entrapment could cause symptoms similar to those mentioned in the article. Neurologic evaluation, including electromyography and a nerve conduction test, would prove diagnostically beneficial.

Similarly, a clinical test that anesthetically blocks the supras-

capular nerve and produces symptom relief would also be indicative.

With either diagnosis, osteopathic manipulative treatment would certainly be appropriate.

\section{JAMES H.CORETTI, DO \\ Director, \\ Work Fitness Clinic \\ Grand Rapids, Mich}

\section{Response:}

\section{To the Editor:}

Repetition strain injury of the supraspinatus muscle responds well to injection delivered at the trigger point. Dexamethasone acetate $(1.5 \mathrm{~mL})$ with Marcaine (1 $\mathrm{mL}$ ) injected by use of a 22gauge, 1.5-inch needle is effective. When such conservative care proves ineffective, or history and physical examination warrant it, further diagnostic studies are indicated.

Carpal tunnel syndrome and a herniated disc at C5 - C6 with osteophytic spurs and resultant spinal stenosis are examples of entrapment neuropathies that can cause a similar clinical picture. Results of neurologic examination, electromyography, and a nerve conduction test may be normal. In such instances, magnetic resonance imaging or contrastenhanced computed tomography, preceded by myelography, may be necessary.

Regarding the neurologic portion of Dr Coretti's letter, my colleagues and I consulted a boardcertified neurologist. In his opinion, injury to the suprascapular nerve would occur with a history of direct macrotrauma to this re- gion, such as a gunshot wound. Complete loss of external rotation and supraspinatus and infraspinatus muscle mass atrophy would occur. Finally, because of this nerve's deep anatomic location, electromyography and a nerve conduction test would be difficult and may produce inaccurate results.

EDWARD JACOBSON, DO Pinellas Park, Fla

\section{Correction to September JAOA}

On page 1148 of the article, "Child sexual abuse: A physician's introduction to historical and medical validation," by Martin A. Finkel, DO, there is an error in the text of the second bulleted point under the subheading "Sexually transmitted diseases." In the third sentence, JAOA inadvertently reversed the mediums used for Chlamydia and for Neisseria gonorrhoeae. The text should read:

For Chlamydia, only cell culture in a proper medium is appropriate. For Neisseria gonorrhoeae species identification, an appropriate medium such as modified Thayer-Martin in an enriched carbon dioxide environment should be used for specimen collection. 